\title{
Improved Maximum a Posteriori Cortical Segmentation by Iterative Relaxation of Priors
}

\author{
Manuel Jorge Cardoso ${ }^{1}$, Matthew J. Clarkson ${ }^{1,2}$, Gerard R. Ridgway ${ }^{1,2}$, \\ Marc Modat ${ }^{1}$, Nick C. Fox ${ }^{2}$, and Sebastien Ourselin ${ }^{1,2}$ \\ 1 Centre for Medical Image Computing (CMIC), University College London, UK \\ ${ }^{2}$ Dementia Research Centre (DRC), University College London, UK
}

\begin{abstract}
Thickness measurements of the cerebral cortex can aid diagnosis and provide valuable information about the temporal evolution of several diseases such as Alzheimer's, Huntington's, Schizophrenia, as well as normal ageing. The presence of deep sulci and 'collapsed gyri' (caused by the loss of tissue in patients with neurodegenerative diseases) complicates the tissue segmentation due to partial volume (PV) effects and limited resolution of MRI. We extend existing work to improve the segmentation and thickness estimation in a single framework. We model the PV effect using a maximum a posteriori approach with novel iterative modification of the prior information to enhance deep sulci and gyri delineation. We use a voxel based approach to estimate thickness using the Laplace equation within a Lagrangian-Eulerian framework leading to sub-voxel accuracy. Experiments performed on a new digital phantom and on clinical Alzheimer's disease MR images show improvements in both accuracy and robustness of the thickness measurements, as well as a reduction of errors in deep sulci and collapsed gyri.
\end{abstract}

\section{Introduction}

Automatic thickness measurements of the cerebral cortex from magnetic resonance imaging (MRI) can aid diagnosis and provide valuable information about the temporal evolution of several diseases. Several surface [1] and voxel-based [2:344 approaches have been proposed. Although surface based approaches allow easier inter-subject thickness comparisons they are computationally very demanding, often requiring laborious manual interaction at several stages. In contrast, voxel based approaches are much more computationally efficient but are also more prone to noise and partial volume (PV) effects. The presence of PV effect in collapsed grey matter folds leads to the existence of PV-corrupted deep sulci and collapsed gyri, the latter mainly caused by the loss of white matter in patients with neurodegenerative diseases.

Several methods have been used to segment the brain into its different structures. Expectation-Maximisation (EM) based algorithms proposed by Wells et al. [5], Van Leemput et al. 6] and Ashburner and Friston 7] are among the most popular and accurate 8 . Prior information about the brain anatomy is generally used to initialise and locally constrain EM based segmentation algorithms, 

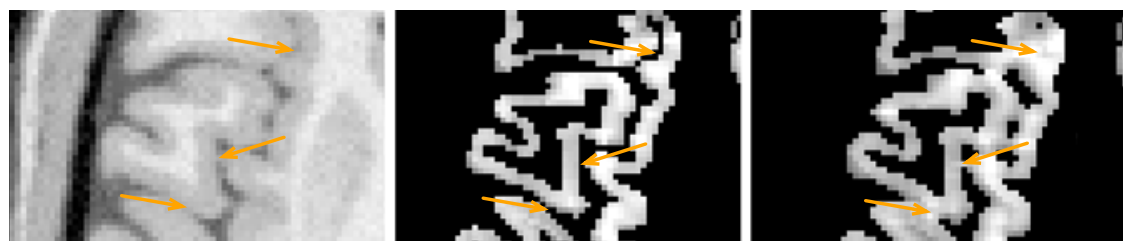

Fig. 1. BrainWeb thickness measurements: Left) BrainWeb image (noise 3\%, INU 20\%); Centre) Proposed method; Right) MAP with MRF but without the proposed improvements. The orange arrows point to areas of improved delineation with the proposed method.

increasing the robustness to noise. However, in some cases, due to intensity nonuniformity (INU), PV and noise, the local difference in intensity is insufficient to provide a correct segmentation of fine structures. The use of priors may also cause problems in areas that have some degree of natural variability, as the prior information used is representative of a normal population and not of the particular subject under study. All these problems lead to an incorrect delineation of collapsed grey matter folds, resulting in incorrect thickness estimates. Acosta et al. 4], used information derived from an Euclidean distance tranform to modify the cost function of a Markov Random Field (MRF) and added a post processing step to solve this problem. However, the use of an ad-hoc mask produced by a distance-ordered homotopic thinning (DOHT) algorithm that binarily unassigns voxels from the thickness calculation may lead to an erratic solution. Hutton et al. 2] used a mathematical morphology based layering method to detect deep sulci, without taking the PV effect or the intensity of the voxels into account, resulting in a loss of accuracy. Additionally, both approaches are only concerned with improvements in the delineation of deep sulci. However, incorrect thickness estimates can also result from loss of tissue in the gyri, which together with PV effects and structural readjustments can lead to a collapsed gyri.

We propose a unified Maximum a Posteriori (MAP) based framework that iteratively changes the priors, improving the PV classification and the delineation of deep sulci and gyri (Fig 1). Both the solution of the EM algorithm and the information derived from the Euclidean distance are used to locally modify the priors and the weighting of the MRF, enabling the detection of small variations in intensity while maintaining robustness of noise. Because of the MRF, the thickness of the PV layer is reduced, making it more in line with the theoretical anatomical limit. This obviates the need for an empirical threshold or distance to stop the search for the correct border within the PV classified area. After the convergence of the MAP algorithm, the cortical thickness is computed using an Eulerian-Lagrangian approach, as in Acosta et al. 44.

\section{Method}

\subsection{Intensity Model and MRF Regularization}

Starting from the image model developed by Van Leemput et al. [6], let $y=\left\{y_{1}\right.$, $\left.y_{2}, \ldots, y_{n}\right\}$, denote the intensities of an MR image of size $n$. Let $z=\left\{z_{1}, z_{2}, \ldots z_{n}\right\}$ 
denote the tissue type to which voxel $i$ belongs. For $K$ tissue types, let $z_{i}=e_{k}$ for some $\mathrm{k}, 1 \leq k \leq K$ where $e_{k}$ is a unit vector with the $k$ th component equal to one and all the other components equal to zero.

Additionally, consider that a bias field can be represented as a linear combination $\sum_{j} c_{j} \phi_{j}$ of $J$ smoothly varying basis functions $\phi_{j}(x)$, with $1 \leq j \leq J$ and $x$ denotes the spatial position, and $C=\left\{c_{1}, c_{2}, \ldots, c_{j}\right\}$ denote the bias field parameters. Let $\Phi_{y}=\left\{\theta_{1}, \theta_{2}, \ldots, \theta_{K}, C\right\}$ represent the overall model parameters. Due to the multiplicative nature of the MR bias field, log-transformed intensities are used, making the bias field additive. Now suppose that the intensity of the voxels that belong to class $k$ are log-normal distributed with mean $\mu_{k}$ and variance $\sigma_{k}^{2}$ grouped in $\theta_{k}=\left\{\mu_{k}, \sigma_{k}^{2}\right\}$. The probability density that voxel $i$ with intensity $y_{i}$ belongs to class $k$ is then

$$
f\left(y_{i} \mid z_{i}=e_{k}, \Phi_{y}\right)=G_{\sigma_{k}}\left(y_{i}-\mu_{k}-\sum_{j} c_{j} \phi_{j}\left(x_{i}\right)\right)
$$

where $G_{\sigma_{k}}$ ( ) denotes a zero-mean normal distribution with variance $\sigma_{k}^{2}$.

By applying the EM algorithm, the Maximum Likelihood (ML) of the model parameter $\Phi_{y}$ provides the following equations:

$$
\begin{gathered}
p_{i k}^{(m+1)}=\frac{f\left(y_{i} \mid z_{i}=e_{k}, \Phi_{y}^{(m)}\right) f\left(z_{i}=e_{i}\right)}{\sum_{j=1}^{K} f\left(y_{i} \mid z_{i}=e_{k}, \Phi_{y}^{(m)}\right) f\left(z_{i}=e_{i}\right)} \\
\mu_{k}^{(m+1)}=\frac{\sum_{i=1}^{n} p_{i k}^{(m+1)}\left(y_{i}-\sum_{j=1}^{J} c_{j}^{(m)} \phi_{j}\left(x_{i}\right)\right)}{\sum_{i=1}^{n} p_{i k}^{(m+1)}} \\
\left(\sigma_{k}^{(m+1)}\right)^{2}=\frac{\sum_{i=1}^{n} p_{i k}^{(m+1)}\left(y_{i}-\mu_{k}^{(m+1)}-\sum_{j=1}^{J} c_{j}^{(m)} \phi_{j}\left(x_{i}\right)\right)^{2}}{\sum_{i=1}^{n} p_{i k}^{(m+1)}}
\end{gathered}
$$

where $m$ denotes the number of iterations. The estimation of $c_{j}^{(m+1)}$ is provided by Van Leemput et al. [6].

Instead of a ML type approach, we adapted the model to a MAP approach by incorporating prior probability information derived from digital brain atlas. These atlases are brought into correspondence using an affine registration [9] followed by a free-form non-rigid registration algorithm [10. The prior probability is introduced as a weight $\pi_{i k}=\left\{\pi_{i 1}, \pi_{i 2}, \pi_{i 3}\right\}$, where $\pi_{i 1}, \pi_{i 2}$ and $\pi_{i 3}$ contain the digital atlas prior probability of white matter (WM), grey matter (GM) and cerebrospinal fluid (CSF) respectively and are integrated in equation 1 as

$$
f\left(y_{i} \mid z_{i}=e_{k}, \Phi_{y}\right)=\pi_{i k} G_{\sigma_{k}}\left(y_{i}-\mu_{k}-\sum_{j} c_{j} \phi_{j}\left(x_{i}\right)\right)
$$

Equations 2, 3] and 4 remain valid and the initial values for $p_{i k}^{0}, \mu_{k}^{0}$ and $\sigma_{k}^{0}$ are given by their equations with $c_{j}^{(0)}=0$ and $f\left(y_{i} \mid z_{i}=e_{k}, \Phi_{y}^{0}\right)=\pi_{i k}$. 
Unfortunately, the intensity model alone only works in relatively ideal conditions because it only classifies the voxels of the image based on the intensity and the initial prior information. Therefore, the model has to be made more robust to noise by including spatial constraints derived from the anatomical properties of the tissues. This is achieved by the use of an MRF that assumes the probability that voxel $i$ belongs to tissue $k$ depends on its neighbours. Using the same approximation as described in [6], Equation 2 will now be

$$
p_{i k}^{(m+1)}=\frac{f\left(y_{i} \mid z_{i}=e_{k}, \Phi_{y}^{(m+1)}\right) f\left(z_{i}=e_{k} \mid p_{\mathcal{N}_{i}}^{(m)} \Phi_{z}^{(m)}\right)}{\sum_{j=1}^{K} f\left(y_{i} \mid z_{i}=e_{k}, \Phi_{y}^{(m+1)}\right) f\left(z_{i}=e_{k} \mid p_{\mathcal{N}_{i}}^{(m)} \Phi_{z}^{(m)}\right)}
$$

with,

$$
f\left(z_{i}=e_{k} \mid p_{\mathcal{N}_{i}}^{(m)} \Phi_{z}^{(m)}\right)=\frac{e^{-\beta U_{\mathrm{mrf}}\left(z_{i} \mid p_{\mathcal{N}_{i}}^{(m)}, \Phi_{z}^{(m)}\right)}}{\sum_{j=1}^{K} e^{\left.-\beta U_{\mathrm{mrf}}\left(z_{i} \mid p_{\mathcal{N}_{i}}^{(m}\right), \Phi_{z}^{(m)}\right)}}
$$

where $U_{\operatorname{mrf}}\left(z_{i} \mid p_{\mathcal{N}_{i}}, \Phi_{z}\right)$ is an energy function dependant on the parameters $\Phi_{z}=\{G, H\} . G$ and $H$ are $K \times K$ matrixes that control the energy of the transition between classes, and $p_{\mathcal{N}_{i}}$ is the value of $p$ in the 6 nearest neighbours $\mathcal{N}_{i}=\left\{i^{n}, i^{s}, i^{e}, i^{w}, i^{t}, i^{b}\right\}$. At this stage $\beta$ is constant and equal to 1 . Please refer to Van Leemput et al. [6] for the estimation of $U_{\operatorname{mrf}}\left(z_{i} \mid p_{\mathcal{N}_{i}}, \Phi_{z}\right)$.

\subsection{Prior Probability Relaxation}

The EM algorithm is known to converge to a local optimum. In a MAP approach, the prior probability drives the EM algorithm to a sensible solution, making it more robust to noise and INU. However, in areas with high anatomical variability, the MAP approach can lead to an erroneous solution because the prior probability might be too low to allow the EM to converge to the expected solution. It can also bias the segmentation towards the template, possibly overshadowing some anatomical differences. We propose a method where the prior probability is changed iteratively at each convergence of the EM algorithm, in an anatomically coherent way. As our model parameters become closer to the true solution, we are able to locally relax our prior probability without loosing robustness to noise, INU and PV.

Initially we model the problem with 3 classes, $\{\mathrm{WM}, \mathrm{GM}, \mathrm{CSF}\}$. The prior probability of WM, GM and CSF are derived from an anatomical brain atlas and non-brain structures are removed. After the convergence of the EM algorithm, the model parameters $\Phi_{y}$ are closer to the true solution, even though the structures in areas with low prior probability might not converge to the correct solution. Once the model parameters are closer to the true solution, the priors are relaxed by letting neighbouring classes share prior probability. The updated prior probability after the first convergence of the EM algorithm will be

$$
\pi_{i k}=\left\{p_{i 1}+\alpha p_{i 2}, p_{i 2}+\alpha\left(p_{i 1}+p_{i 3}\right), p_{i 3}+\alpha p_{i 2}\right\} / \Pi_{i}
$$


where $\alpha$ is a pre-specified parameter that controls the percentage of prior probability sharing (set to 0.2 here) and $\Pi_{i}$ is a normalisation constant ensuring $\sum_{k=1}^{K} \pi_{i k}=1$.

After the second convergence of the EM algorithm, we use the values of $p_{i k}$, $\mu_{k}, \sigma_{k}$ to initialise a 5 class model, that considers 3 pure tissue classes and 2 mixture classes $\{\mathrm{WM}, \mathrm{GM}, \mathrm{CSF}, \mathrm{WM} / \mathrm{GM}, \mathrm{GM} / \mathrm{CSF}\}$. All the classes are modelled as Gaussian mixtures in the same framework as before. The prior probability, average and variance for the 5 class model are denoted as $\pi_{i k}^{*}, \mu_{k}^{*}$ and $\left(\sigma_{k}^{2}\right)^{*}$, where the superscript ${ }^{*}$ is used to indicate that they belong to the 5 class model. They are initialised as

$$
\begin{gathered}
\pi_{i k}^{*}=\left\{p_{i 1}, p_{i 2}, p_{i 3}, \sqrt{p_{i 1}^{*} p_{i 2}}, \sqrt{p_{i 2}^{*} p_{i 3}}\right\} / \Pi_{i} \\
\mu_{k}^{*}=\left\{\mu_{1}, \mu_{2}, \mu_{3}, \Gamma_{1 / 2} \mu_{1}+\left(1-\Gamma_{1 / 2}\right) \mu_{2}, \Gamma_{2 / 3} \mu_{2}+\left(1-\Gamma_{2 / 3}\right) \mu_{3}\right\} \\
\left(\sigma_{k}^{2}\right)^{*}=\left\{\sigma_{1}^{2}, \sigma_{2}^{2}, \sigma_{3}^{2}, \Gamma_{1 / 2}^{2} \sigma_{1}^{2}+\left(1-\Gamma_{1 / 2}^{2}\right) \sigma_{2}^{2}, \Gamma_{1 / 2}^{2} \sigma_{2}^{2}+\left(1-\Gamma_{1 / 2}^{2}\right) \sigma_{3}^{2}\right\}
\end{gathered}
$$

where $\Pi_{i}$ is a normalisation constant over $k$ and $\Gamma_{j / k}$ is the average of the fractional content (FC) between classes $j$ and $k$, excluding values of FC outside $[0,1]$, where FC is defined as $F C=\left(\mu_{j}-\bar{y}_{i}\right) /\left(\mu_{j}-\mu_{k}\right)$ and $\bar{y}_{i}=y_{i}-\sum_{j} c_{j} \phi_{j}\left(x_{i}\right)$ is the INU corrected intensity. This new stage of the EM algorithm is initialised with $c_{j}^{*}=c_{j}$ and $f\left(y_{i} \mid z_{i}=e_{k}, \Phi_{y}\right)=\pi_{i k}^{*}$.

\subsection{Deep Sulci and Gyri Delineation}

After the EM algorithm converges again, due to the presence of the MRF, fine structures such as deep sulci and gyri might not be correctly segmented. In Van Leemput et al. [6], the super- and sub-diagonal of the matrices $\mathrm{G}$ and $\mathrm{H}$ are constrained to be equal to the diagonal itself, i.e., $G_{(i, i)}=G_{(i, i+1)}=G_{(i, i-1)}$ and $H_{(i, i)}=H_{(i, i+1)}=H_{(i, i-1)}$. This type of constraint helps the detection of fine structures, however it globally makes the segmentation less robust to noise. To overcome this limitation, we propose a method to locally weight the MRF algorithm and relax the prior probability. This way, the MRF can still be robust to noise and simultaneously allow the segmentation and correct labelling of fine structures. In a similar way to [4], we use the information derived from a Euclidian distance transform to estimate the location of deep sulci and gyri and change the priors and the weighting of the MRF only in those locations. The functions $\omega_{i}^{\text {gyri }}, \omega_{i}^{\text {sulci }}$ that are used to relax the priors are defined as follows:

$$
\begin{gathered}
\omega_{i}^{\text {gyri }}=\log \left(p_{i \mathrm{GM}_{0.5}^{*}}^{*}\left(1-\left\|\nabla E_{i}^{\mathrm{CSF}+}\right\|\left(1-p_{i \mathrm{CSF}}^{*}-p_{i \mathrm{GM} / \mathrm{CSF}}^{*}\right)\right)+1\right) / \Omega^{\text {gyri }} \\
\omega_{i}^{\text {sulci }}=\log \left(p_{i \mathrm{GM}_{0.5}^{*}}^{*}\left(1-\left\|\nabla E_{i}^{\mathrm{WM}+}\right\|\left(1-p_{i \mathrm{WM}}^{*}-p_{i}^{*} \mathrm{WM} / \mathrm{GM}\right)\right)+1\right) / \Omega^{\text {sulci }}
\end{gathered}
$$

where the $\Omega$ are normalisation factors, $E_{i}^{\mathrm{WM}+}$ is the distance to the sum of WM and WM/GM labelled areas thresholded at 0.5 (and similarly for $E_{i}^{\mathrm{CSF}+}$ with 
CSF and GM/CSF), and $p_{\mathrm{GM}_{0.5}}^{*}$ is $p_{\mathrm{GM}}^{*}$ also thresholded at 0.5. The weighting of the $\mathrm{MRF}$ is incorporated in Equation 7 by replacing $\beta$ with a spatially-varying value

$$
\beta_{i}=\left(\left(1-\omega_{i}^{\text {sulci }}\right)\left(1-\omega_{i}^{\text {gyri }}\right)\right) / \Omega^{\beta}
$$

The values of $\omega^{\text {sulci }}$ and $\omega^{\text {gyri }}$ vary between $[0,1]$ and have a value of 1 near the centre of the sulci and the centre of the gyri respectively. In a same way, the value of $\beta_{i}$ is normalized by $\Omega^{\beta}$ to lie between $[0,1]$ and has a value of 0 near the centre of the sulci and gyri. The functions $\omega_{i}^{\text {sulci }}$ and $\omega_{i}^{\text {gyri }}$ are going to be used to iteratively relax $\pi_{i k}$ to give more prior probability to the respective mixture classes in areas where deep sulci and gyri should exist. $\pi_{i k}$ is updated as

$$
\pi_{i k}^{*}=\left\{p_{i 1}, p_{i 2}, p_{i 3}, p_{i 4}+\omega_{i}^{\text {gyri }} p_{i 2}, p_{i 5}+\omega_{i}^{\text {sulci }} p_{i 2}\right\} / \Pi_{i}
$$

This last EM stage is iterated several times and every time the EM converges, $\omega_{i}^{\text {sulci }}, \omega_{i}^{\text {gyri }}, \beta_{i}$ and $\pi_{i k}^{*}$ are updated, and a new EM starts. The algorithm finishes when the change in $p_{i k}^{*}$ at successive converged values of the EM algorithm is less than a predefined $\varepsilon$.

\subsection{Thickness Calculation}

The cortical thickness is then computed using a hybrid Lagrangian-Eulerian approach to solve the Laplace equation, as in [4. This method takes into account the PV effect and greatly improves the thickness results. The final values of $p_{i k}$ are used to create the labelled image, where each voxel is set to the most probable tissue. The grey matter fractional content image, used in the thickness calculation algorithm, is set to 1 for every voxel belonging to pure GM and set to the correspondent $\mathrm{FC}$ for voxels belonging to mixture classes.

\section{Experiments and Results}

We created a very high resolution phantom containing finger and sheet like collapsed sulci and gyri. The Euclidean thickness of the structure is constant and equal to 8 . This leads to an average Laplace equation based thickness of 8.13 and a standard deviation of 0.15 measured in the high resolution phantom. We then use Fourier-resampling to reduce the resolution by a factor of 5 , before adding complex Gaussian noise (either low or high level) and taking the magnitude, resulting in two low resolution Rician noise corrupted phantoms. To obtain artificial priors, the ground truth image was Gaussian filtered $(\sigma=4 \mathrm{~mm})$ to simulate the anatomical variability.

The results are shown in Fig 2. The average (standard deviation) thickness using the proposed method is $8.36(0.44)$ and $8.76(0.77)$ for the low and high noise phantom respectively. These values are in line with the expected value of 8.13(0.15). The MAP approach with the MRF but without the proposed improvements yields an average of 12.87 (2.98) and 12.49 (2.82) for the low and high noise phantom respectively, values of thickness much higher than expected 

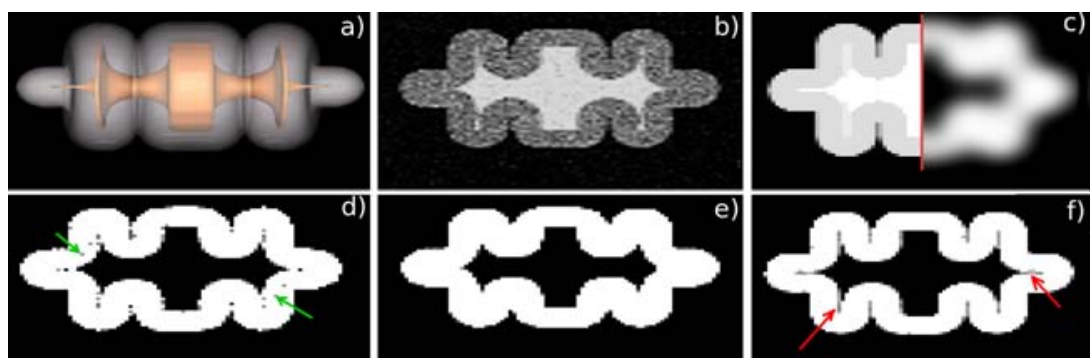

Fig. 2. Phantom segmentation for thickness: a) 3D model of the phantom, b) High noise phantom, c) True labels and GM prior used, d) ML without MRF, e) ML with MRF, f) Proposed method. The green arrows point to the presence of noise causing wrong thickness measurements. The red arrows point to the detected deep gyri.

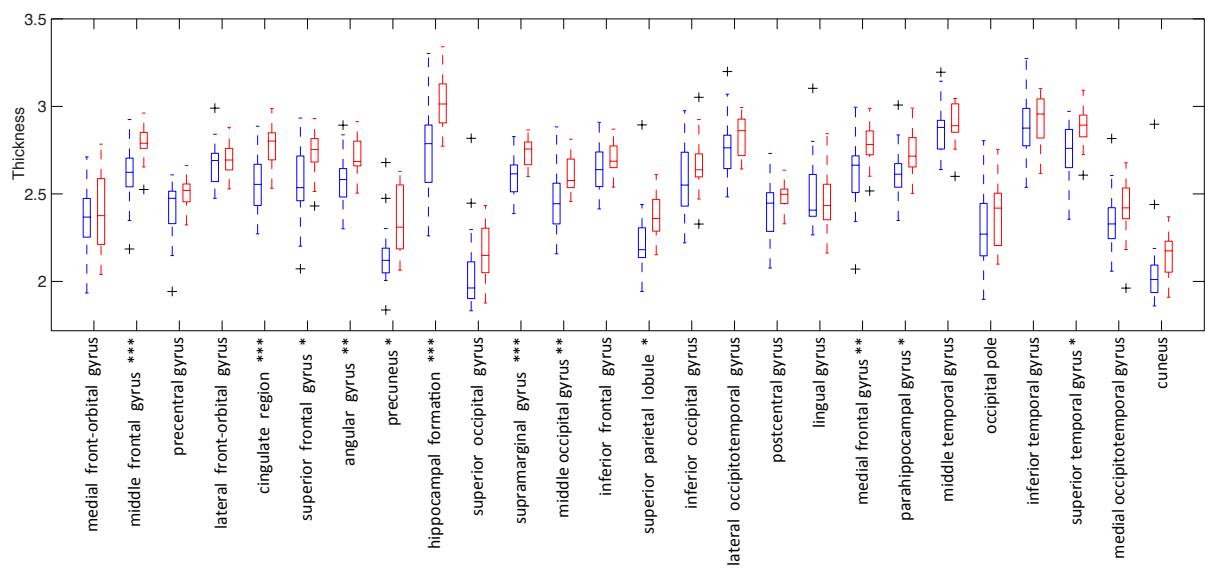

Fig. 3. Box plot of the distribution for all AAL regions over all patients (blue/left) and controls (red/right). The symbols $*, * *$ and $* * *$ correspond to areas with a significance level of $\mathrm{p}<0.01,0.001$ and 0.0001 respectively.

due to the mis-detection of the deep sulci and gyri. Finally, the approach without either the MRF or the proposed improvements yielded an average of 12.11 (2.55) and 9.35 (3.1) for the low and high noise phantom respectively. The average thickness for the high noise phantom using this last technique is closer to the true value of 8 than for the the low noise phantom, but this is due to the noise introduced by the lack of MRF leading to a number of short paths to mis-segmented voxels.

Secondly we tested our method on real data, comprising 28 Alzheimer's disease (AD) patients and 17 age- and gender-matched controls. T1-weighted volumetric images were aquired on a $1.5 \mathrm{~T}$ Signa unit (GE Medical Systems, Milwaukee, WI) with 256x256 in-plane resolution, and 124 contiguous $1.5 \mathrm{~mm}$ coronal slices were acquired using a spoiled fast GRASS sequence $(\mathrm{TR}=15 \mathrm{~ms}$; TE $=5.4 \mathrm{~ms}$; flip angle $=15 ; \mathrm{TI}=650 \mathrm{~ms}$ ). 
Further details of the protocol and subject characteristics can be found in 11. The same transformation used to map the priors to the individual subjects was used to propagate the AAL template [12, and average thickness values were computed over 26 bilateral AAL regions. Fig 3 shows the distribution, at baseline, of values over patients and controls, illustrating group separation in the different brain regions. To statistically quantify the group-separation, we performed two-tailed unequal-variance two-group t-tests over all the AAL regions; significance is indicated in the legend of Fig 3. The best group separation was acheived in two of the regions (cingulate and hippocampal formation) known to be severely affected in AD.

\section{Conclusions}

We present an extension of previous work to improve the accuracy of cortical thickness measurements by refining and enhancing the segmentation of the cortex. The main contribution of this work lies in a method that iteratively relaxes and modifies the prior information in an anatomically coherent way to ameliorate the key problem of PV effect and reduce the bias towards the priors.

The method achieves better delineation of collapsed grey matter folds without loosing robustness to noise and intensity inhomogeneity. All segmentation steps (such as the transition from pure-tissue to PV model) are encompassed in a single framework, without ad-hoc post-processing. Quantitative analysis of a phantom using the proposed method demonstrated improvements in the accuracy and robustness of the thickness calculation when compared to other methods. Results on real data showed clinically-expected patterns of cortical thickness in AD, with highly significant group differences in several areas.

In the future, we plan to expand and improve our technique to study the temporal evolution of cortical thickness in neurodegenerative diseases.

\section{References}

1. Fischl, B., Dale, A.: Measuring the thickness of the human cerebral cortex from magnetic resonance images. PNAS 97, 11044-11049 (2000)

2. Hutton, C., Vita, E.D., Ashburner, J., Deichmann, R., Turner, R.: Voxel-based cortical thickness measurements in MRI. Neuroimage 40, 1701-1710 (2008)

3. Lohmann, G., Preul, C., Hund-Georgiadis, M.: Morphology-based cortical thickness estimation. In: IPMI 2003, pp. 89-100 (2003)

4. Acosta, O., Bourgeat, P., Fripp, J., Bonner, E., Ourselin, S., Salvado, O.: Automatic delineation of sulci and improved partial volume classification for accurate 3D voxel-based cortical thickness estimation from MR. In: Metaxas, D., Axel, L., Fichtinger, G., Székely, G. (eds.) MICCAI 2008, Part I. LNCS, vol. 5241, pp. 253261. Springer, Heidelberg (2008)

5. Wells, M., Grimson, W.E.L., Kikinis, R., Jolesz, F.A.: Adaptive segmentation of MRI data. IEEE Transactions on Medical Imaging 15, 429-442 (1996)

6. Van Leemput, K., Maes, F., Vandermeulen, D., Suetens, P.: Automated modelbased tissue classification of MR images of the brain. IEEE Transactions on Medical Imaging 18, 897-908 (1999) 
7. Ashburner, J., Friston, K.: Unified segmentation. Neuroimage 26, 839-851 (2005)

8. Klauschen, F., Goldman, A., Barra, V., Meyer-Lindenberg, A., Lundervold, A.: Evaluation of automated brain MR image segmentation and volumetry methods. Human Brain Mapping 30, 1310-1327 (2009)

9. Smith, S., Jenkinson, M., Woolrich, M., Beckmann, C., Behrens, T., Bannister, P., DeLuca, M., Drobnjak, I., Flitney, D.: Advances in functional and structural MR image analysis and implementation as FSL. Neuroimage 23, 208-219 (2004)

10. Modat, M., Taylor, Z.A., Barnes, J., Hawkes, D.J., Fox, N.C., Ourselin, S.: Fast free-form deformation using the normalised mutual information gradient and graphics processing units. In: High-Performance MICCAI workshop (2008)

11. Schott, J., Price, S., Frost, C., Rossor, M., Fox, N.: Measuring atrophy in Alzheimer disease: a serial MRI study over 6 and 12 months. Neurology 65(1), 119-124 (2005)

12. Tzourio-Mazoyer, N., Landeau, B., Crivello, F., Etard, O., Delcroix, N., Mazoyer, B., Joliot, M.: Automated anatomical labeling of activations in SPM using a macroscopic anatomical parcellation of the MNI. Neuroimage 15, 273-289 (2002) 